\title{
Newer MR Imaging Techniques in Traumatic Brachial Plexopathies
}

\author{
Amit Disawal ${ }^{1^{\star}}$, Ashwini Bakde² \\ ${ }^{1}$ Associate Professor, ${ }^{2}$ Assistant Professor, \\ Department of Radiodiagnosis, Government Medical College and Hospital, Nagpur, India.
}

\begin{abstract}
Introduction: The aim of this study was to identify, localize and characterize various traumatic brachial plexus pathologies with the help of newer MR techniques. MRI enables the differentiation of preganglionic from postganglionic injuries, a distinction that is critical in management decisions.

Materials \& Methods: 34 patients presenting with traction injuries of the brachial plexus were included in the study. T1W and $\mathrm{T} 2 \mathrm{~W}$ coronal, sagittal and axial images of the cervical spine and oblique coronal images of the brachial plexus were obtained with $2 \mathrm{~mm}$ thickness with Diffusion Weighted Imaging with Background Suppression and Fat saturation Contrast Enhanced T1 Weighted transverse and oblique-coronal scan. Ethical committee clearance for the study was obtained.

Results: The results were classified into 4 major categories (normal rootlet, rootlet injuries, avulsion, and meningocele).The results were compared with surgical findings, intraoperative neurophysiology and subsequent clinical progress. Surgery for exploration and repair have been performed in 30 of 34 patients scanned. Evidence of root avulsion was seen in 24 patients. There were no false positives. MRI underestimated the number of individual roots avulsed; sensitivity was $94 \%$. Post-ganglionic injuries were seen in 8 cases. 4 patients, in whom T1WI and T2WI were normal, were found to have
\end{abstract}

\section{INTRODUCTION}

Brachial plexus is a network of nerves located in the neck and axilla formed by the union of the anterior divisions of the lower four cervical nerves and the greater part of the anterior division of the first thoracic nerve; the fourth cervical usually gives a branch to the fifth cervical, and the first thoracic frequently receives one from the second thoracic ${ }^{1}$. This network gives origin to various nerves at various levels which are in order, roots, trunks and cords, which continue further as nerves.

Trauma to brachial plexus is not an uncommon event, and occurs in road traffic accidents, sports injuries, incised wounds to the neck / axilla and various other types of trauma. Clinically brachial plexus injuries can be categorized into upper, lower brachial plexus lesions, posterior cord lesions \& complete brachial plexus injury.

The upper variety injures $\mathrm{C} 5$ and $\mathrm{C} 6$ roots and presents with shoulder and biceps denervation resulting in Erb's palsy (policeman's tip). The lower plexus injury involves $\mathrm{C} 8$ and T1 roots resulting in denervation of forearm flexors and hand small muscles abnormal signal intensities predominantly in post-ganglionic segments on DWIBS. It was possible to define the level of the injury within the plexus. Enhancement of intradural nerve roots and root stumps suggested functional impairment of nerve roots despite morphologic continuity. Abnormal enhancement of paraspinal muscles is an indirect sign of root avulsion injury.

Key Words: Brachial plexus, Traumatic brachial plexopathy, DWIBS (Diffusion Weighted Imaging with Background Suppression), Preganglionic, Postganglionic.

\section{${ }^{*}$ Correspondence to:}

Dr. Amit Disawal (MD),

1157, C.A. Road, Second floor,

Above Dena bank, Gandhibagh, Nagpur, India.

Article History:

Received: 21-08-2016, Revised: 16-09-2016, Accepted: 29-09-2016

\begin{tabular}{|l|c|}
\hline \multicolumn{2}{|c|}{ Access this article online } \\
\hline $\begin{array}{l}\text { Website: } \\
\text { www.ijmrp.com }\end{array}$ & Quick Response code \\
\hline DOI: & \\
10.21276/ijmrp.2016.2.5.050 & \\
\hline
\end{tabular}

of the hand. The posterior cord lesions involve $\mathrm{C} 7$ root and presents with paralysis of triceps, extensors of wrist \& digits.

The aim of this study is to identify, localize and characterize various traumatic brachial plexus pathologies, differentiate between preganglionic and postganglionic injuries and evaluate the role of newer MR techniques - MR Myelography and DWIBS (Diffusion Weighted Imaging with Background Suppression). The differentiation between preganglionic and postganglionic injuries is of utmost clinical importance due to differing management options. Preganglionic nerve damage cannot be repaired, so nerve transfers are used to manage. Postganglionic damage with continuity preservation is given conservative management for spontaneous recovery and if disrupted, nerve grafting is undertaken.

\section{SUBJECTS AND METHODS}

Type of study

Observational prospective study. 


\section{Duration of study}

April 2015 and March 2016.

\section{Inclusion criterion}

34 patients presenting with clinical suspicion / signs of brachial plexus injuries between were included in this study. The age group was between 7 and 48 years.

\section{Exclusion criterion}

Patients with brachial plexopathies due to non-traumatic aetiology were not included in this study, so were patients with contraindications to undergo MRI like patients who had metallic prosthesis, implants, pacemakers etc or who were not willing to consent for the study. Claustrophobic patients were given the option of sedation during scanning in the presence of an expert anaesthesiologist.

Written consent was taken from all patients regarding MRI scanning and willingness for the study. Ethical approval for the study was obtained from institutional ethical committee.

\section{Materials}

The patients were scanned in a 1.5 Tesla MRI machine (Philips MR Systems Achieva, Philips Medical Systems, The Nederlands; 1.5 T MRI machine) with a Wrap Around body surface coil. T1W
(TR [Time of repetition] 600-700, TE [Time to Echo] 20, NSA [Number of Signal Averages] 4) and T2W (TR 1800, TE 90, NSA 2) coronal, sagittal and axial sequences of the cervical spine and oblique coronal sequences of the brachial plexus were performed and in addition, fat-sat CET1 transverse and oblique scans and newer MR sequences - MR Myelography (Single shot TSE,TR 8000, TE1000, TSE factor 256, acquisition time 3:36) and Diffusion Weighted Imaging with Background Suppression (TR5000 , TE- 70 , TI- $180 \mathrm{~ms}$ at 1.5 T, Sense factor- 2 , EPI factor-7 were also obtained.

Injuries were categorized into preganglionic, postganglionic or combined varieties. Specific Zones of brachial plexus were defined to localize lesions to a particular level / zone according to modified Nagano classification of brachial plexus injuries ${ }^{2}$. Zone 1 was preganglionic nerve root avulsion from the spinal cord; zone II-A, root lesion within the intraforminal space; zone II-B, post ganglionic nerve root avulsion distal to the intraforaminal space; zone III, trunk injury and zone IV, injury to cord. The pattern of occurrence, demographic distribution, frequency of magnetic resonance imaging signs using the newer imaging modalities and frequency of types of brachial plexus injuries were then studied.

Table 1: Shows presentation of patients with different levels of brachial plexus injuries

\begin{tabular}{lcc}
\hline Level of injury & Presentation: Signs/symptoms & No. of patients \\
\hline Upper plexus lesions & Paralysis of deltoid, supra/infraspinatus, biceps, biceps, brachioradialis & 13 \\
Posterior cord lesions & Paralysis of triceps, extensor carpi radialis/ulnaris, extensor digitorum & 09 \\
Lower plexus lesions & Sensory loss in distribution of lower trunk & 12 \\
Complete brachial plexus injury & Completely paralyzed arm with no sensation & 0 \\
& & Total Patients 34 \\
\hline
\end{tabular}

Table 2: Common etiologies of brachial plexus injuries

\begin{tabular}{lc}
\hline Aetiology & Number of patients \\
\hline Road traffic accident & $19(55.8 \%)$ \\
Sports injury & $9(26.4 \%)$ \\
Heavy objects & $4(11.7 \%)$ \\
Incised wounds & $2(6 \%)$ \\
\hline
\end{tabular}

Table 3: Shows distribution of nerve root pathologies

\begin{tabular}{llc}
\hline S.NO & Nerve root pathologies & Number of roots \\
\hline 1 & Normal & 76 \\
2 & Partial avulsion & 8 \\
3 & Avulsion & 39 \\
4 & Meningocele & 43 \\
& & 166 \\
\hline
\end{tabular}

Table 4: Frequency of various findings in nerve root injuries

\begin{tabular}{llc}
\hline S.NO & Findings In Nerve Root Injuries & Number \\
\hline 1 & Cord edema & 27 \\
2 & Hemorrhage & 20 \\
3 & Absence of roots & 39 \\
4 & Abnormal muscle enhancement & 22 \\
\hline
\end{tabular}

Table 5: level of involvement in observed brachial plexus injuries

\begin{tabular}{lc}
\hline Level of involvement & Number \\
\hline Root & 82 \\
Trunk & 12 \\
Cord & 3 \\
Nerve & 2 \\
Root and trunk & 12 \\
Root and cord & 3 \\
Root and nerve & 1 \\
\hline
\end{tabular}

\section{RESULTS}

34 patients presenting with clinical suspicion / signs of brachial plexus injuries between were included in this study. Following important observations were made. Patients between 7 and 48 years were part of the study and maximum patients were in their third decade. The study population consisted of predominantly males who constituted $71 \%(n=24)$ and right side was more commonly involved $(n=21,61.7 \%)$.

The most common cause of brachial plexus injury was road traffic accident, with other etiologies as in table 2.

Retrospectively 166 out of 170 nerve roots could be visualized. Four were unclear. The findings in the nerve root level were classified as shown in Table 3.

The findings of root avulsion are cord edema, haemorrhage or scarring within the spinal canal, absence of roots in the intervertebral foramina, and pseudomeningoceles. Their incidences in various root injuries are shown in Table 4.

In the present study, preganglionic injuries were more common than postganglionic injuries with incidence ratio of $3.25: 1$. The various levels of involvement in brachial plexus injuries are shown in Table 5.

There were altogether 114 nerve injuries comprising 90 preganglionic and 24 postganglionic injuries. Conventional MR sequences could identify 76 and 18 injuries out of them, achieving a sensitivity of $84.44 \%$ and $75 \%$ for preganglionic and postganglionic injuries respectively. The newer imaging techniques, MR Myelography and DWIBS (Diffusion Weighted Imaging with whole Body Suppression) on the other hand achieved $100 \%$ sensitivity in diagnosing them with significant statistical superiority over conventional MRI. (Table 6) 
Table 6: Shows sensitivity of conventional MR \& of newer MR sequences in pre \& post-ganglionic brachial plexus injuries.

\begin{tabular}{lcccc}
\hline Category of injury & Number of injuries & $\begin{array}{c}\text { Sensitivity of conventional } \\
\text { MR }\end{array}$ & $\begin{array}{c}\text { Sensitivity of newer } \\
\text { sequences }\end{array}$ & P value \\
\hline Preganglionic & 90 & $84.44 \%$ & $100 \%$ & 0.00009770 \\
Postganglionic & 24 & $75 \%$ & $100 \%$ & 0.00882878 \\
\hline
\end{tabular}

Test used: Chi Square test. Significant: If $p$ value $<0.05$
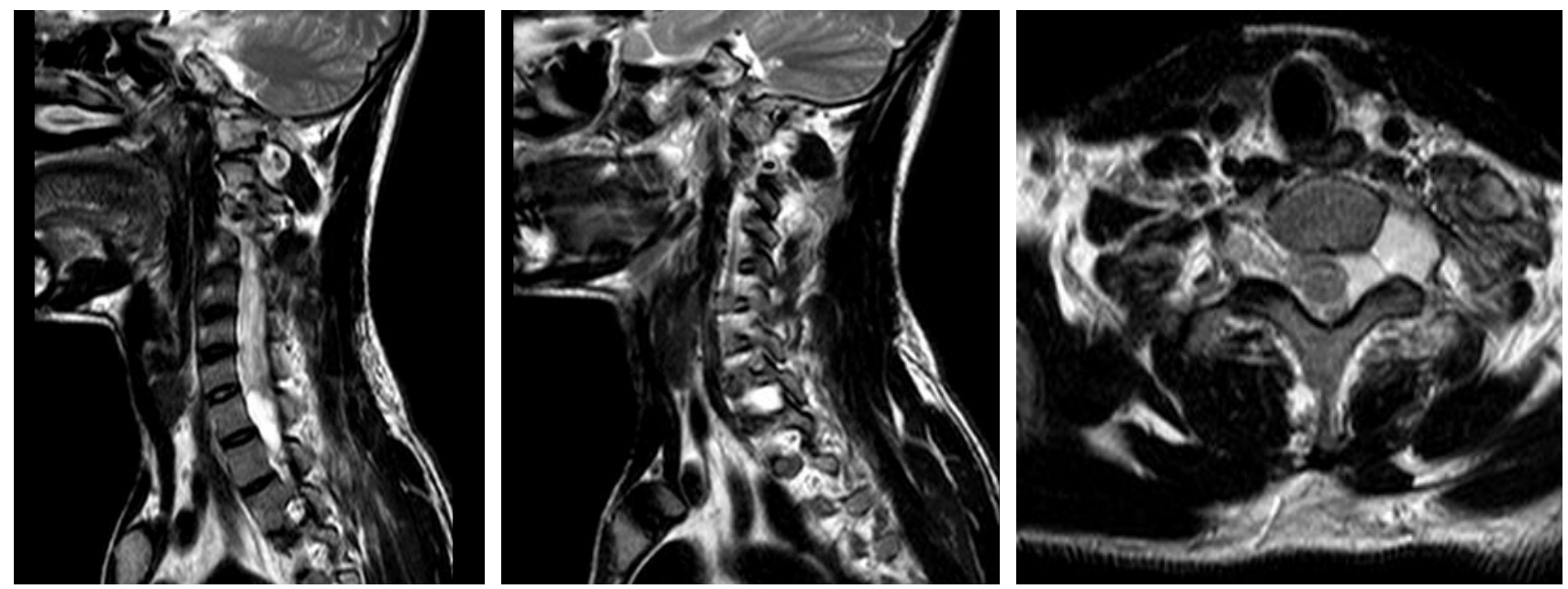

FIG 1A, 1B \& 1C: Sagital T2W Images (1A \& 1B) And Axial T2W Images (1C) Showing Absence of Nerve Roots in Intervertebral Foramina with Meningocele Formation.
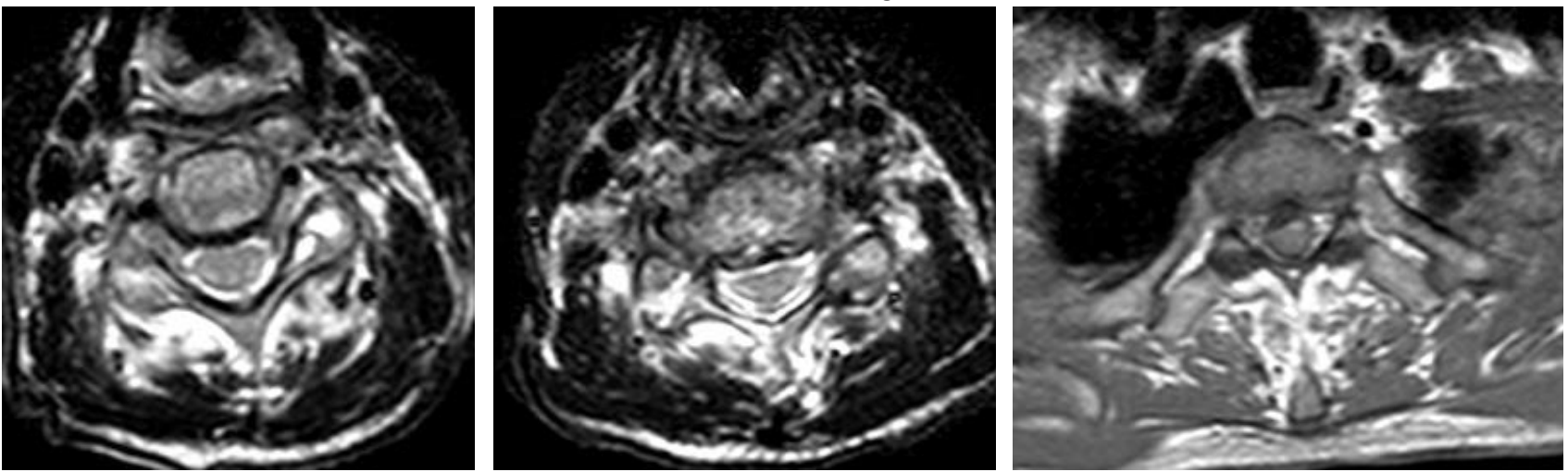

FIG 2A, 2B \& 2C: Axial T2W Images (2A \& 2B) Showing Edema Of The Cord In A 28 Year Old Male Complaining Of Tingling In Right Hand Following Road Traffic Accident. T1W Image (2C) Shows Hyperintensity Suggestive Of Cord Hemorrhage.

\section{DISCUSSION}

Brachial plexus is involved in a variety of primary and secondary neoplastic, traumatic, inflammatory, neuropathic and compressive disorders. Trauma is fairly common and occurs due to undue traction stress on the shoulder in extension or abduction. Trauma can involve any level of brachial plexus. They can be broadly divided into preganglionic and postganglionic lesions. This division is of utmost clinical importance due to differing management options. Preganglionic nerve damage cannot be repaired, so nerve transfers are used in management. Postganglionic damage with continuity preservation is given conservative management for spontaneous recovery and if disrupted, nerve grafting is undertaken.

Nerve root avulsions are the most common category of brachial plexus injury occurring in the preganglionic part. It is caused by simultaneous traction of the arm and lateral flexion of head to the opposite direction, the most common situation to cause such injury being road traffic accident followed by birth injury, fall of heavy object over shoulder and very rarely missile injuries or sharp stab injuries though they are more likely to cause distal plexus injuries. Nerve root avulsions are more commonly accompanied by traumatic pseudomeningocele formation ${ }^{3}$, which can however rarely exist without any nerve root avulsions (Fig 1A, 1B \& 1C). Conversely nerve root avulsions can occur without pseudomeningocele formation. Traumatic pseudomeningoceles are fluid collections in continuity with CSF through the neural foramina. Imaging differentiation between presence and absence of nerve root avulsions with confidence is very important. Computed tomography myelography is considered the gold standard in depicting the nerve roots consistently with anatomic detail $^{4}$. MRI however enjoys the advantages of non-invasiveness in the form of absent necessity to introduce intrathecal contrast, but cannot show with reliability all nerve roots. Hence MRI was of great utilization in visualizing extraforaminal parts of the plexus, with signs of injury being thickening with or without T2 hyperintensity of the plexus components 5 . It can also show causes of plexopathy after trauma like hematoma or fracture of adjacent bone. Conventional MRI can provide some indirect signs of nerve root injury ${ }^{6}$. Signal intensity changes within the spinal cord in the form of acute edema (Fig 2A, 2B \& 2C) with thickened cord with increased signal on $\mathrm{T} 2$ or myelomalacia with thinned $\mathrm{T} 2$ hyperintense cord; and enhancement of nerve roots and 
paraspinal muscles are considered important contributory signs, so is a focus of haemorrhage at the site of origin of root from the cord (Fig 3A, 3B, 4A, 4B). Newer MR sequences like MR myelography and diffusion weighted imaging increase considerably the diagnostic ability of MR in pre and post ganglionic injuries.

MR Myelography (Fig 5A \& 5B) is a heavily T2 weighted 3D sequence providing adequate visualization of nerve roots within the CSF in the spinal canal for confident diagnosis7. Previous studies have documented unreliability of MR myelography in

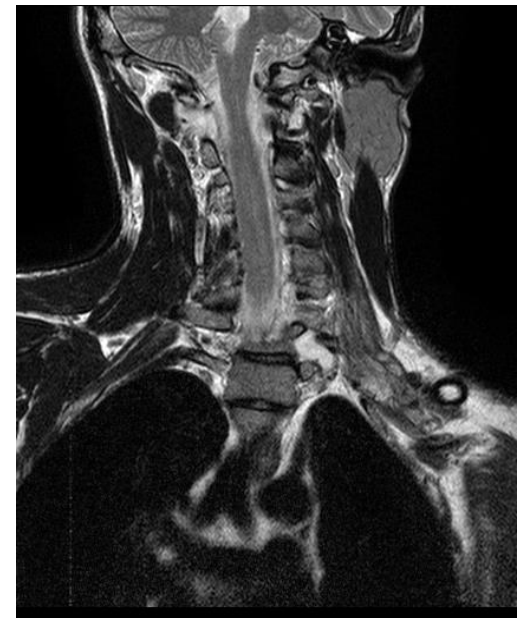

FIG 3A \& 3B: T1 \& T2 Coronal Images Showing Thickened Disrupted Nerves At The C5-C7 Levels On Left Side Suggestive Of Postganglionic Injury Following Trauma.
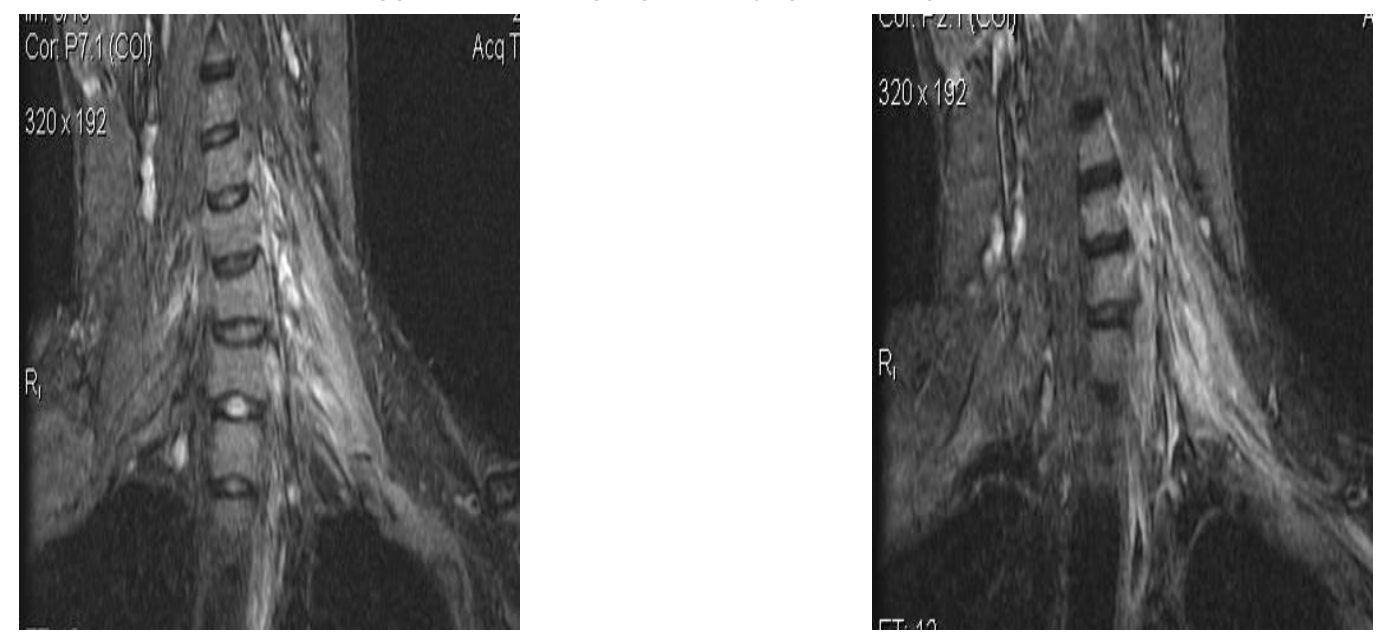

FIG 4A \& 4B: Coronal Fat Suppressed T1W Images Showing Altered Signal Intensity Involving The C5, C6 \& C7 Roots On Left Side With Adjacent Muscle Edema.
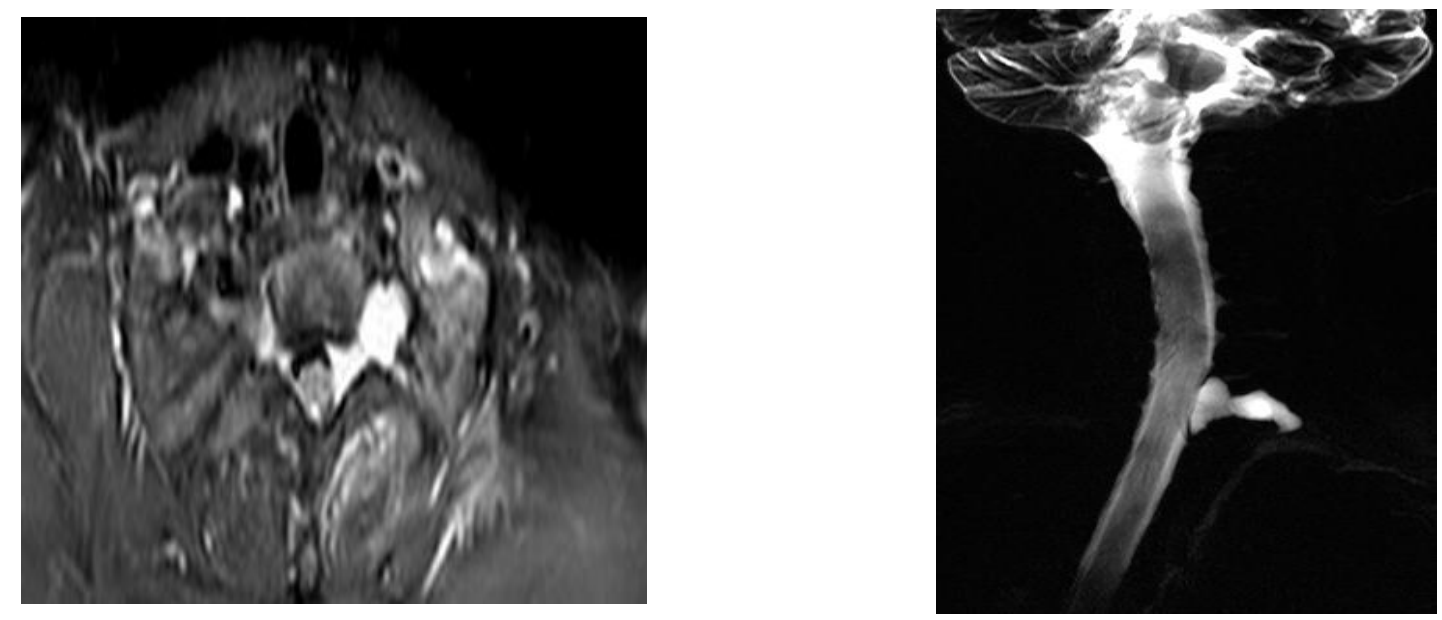

FIG 5A \& 5B: Axial Fat Supressed (5A) And Myelographic Image (5B) Showing Meningocele Formation In A 37 Year Old Patient With Tingling In Left Upper Limb Since 3 Months. 


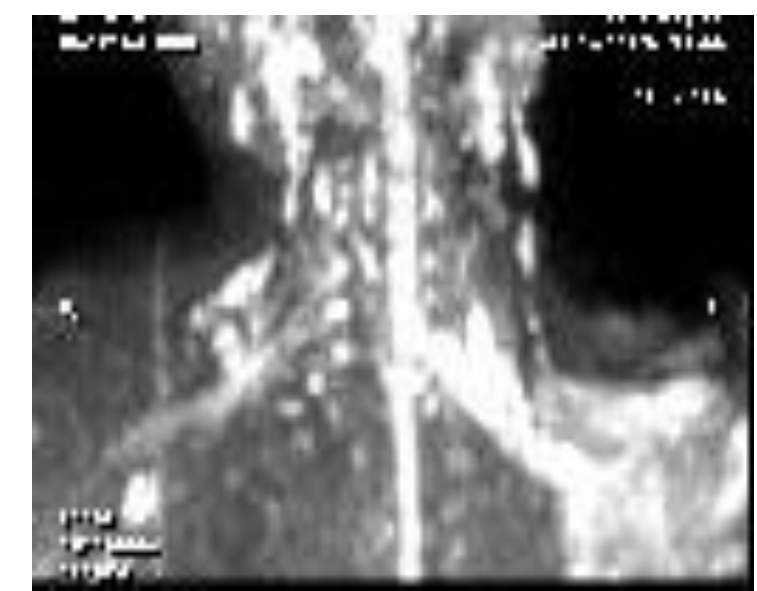

FIG 6: Diffusion Weighted Imaging With Backgound Supression (DWIBS) Shows Non Visualisation Of The Left Sided Nerves. The Avulsed Nerves Are Seen At The Base Of The Left Scalene Triangle.

Diffusion weighted imaging to depict anatomy of neural structures outside the central nervous system is called diffusion weighted neurography. It is similar in diffusion weighted sequences used in body imaging, being different in that it uses a higher $b$ value of around $1000 \mathrm{sec} / \mathrm{mm}^{2}$. STIR (short tau inversion recovery) sequence for fat suppression and free breathing sequence to enable long scanning times are used. Diffusion weighted neurography clearly depicts consistently the postganglionic brachial plexus in healthy subjects and demonstrates loss of continuity in nerve injury with confidence (Fig 6). 3D depiction of the complex anatomy of the post ganglionic roots, trunks and cords facilitate easy appreciation of nerve discontinuity, thickening and comparison with the other side.

\section{CONCLUSIONS}

MRI is crucial to differentiate between preganglionic and postganglionic injuries. It helps to determine the location and severity of injuries more accurately. New techniques including MR Myelography, diffusion-weighted neurography and DWIBS is useful in the accurate evaluation and management of BPI.

DWIBS method with coronal MIP presentation provides an overview image of the brachial plexus with high conspicuity between the nerves and detecting post ganglionic nerve root avulsion while MR Myelography was useful in detecting preganglionic pathologies which were not clearly depicted by conventional MRI.
MR imaging is the only preoperative examination that can help detect functionally impaired nerve roots with anatomic normality and is helpful in avoiding abortive reconstructive procedures ${ }^{8}$.

\section{REFERENCES}

1. Gray, Henry. Anatomy of the Human Body. Philadelphia: Lea \& Febiger, 1918; Bartleby.com, 2000.

2. Nagano A. Treatment of brachial plexus injury .J Orthop Sci 1998:3:71-80.

3. Hayashi N, Yamamoto S, Okubo T, et al. Avulsion injury of cervical nerve roots: enhanced intradural nerve roots at MR imaging. Radiology 1998;206:817-822.

4. Tsuchiya K, Katase S, Aoki C, Hachiya J. Application of multidetector row helical scanning to postmyelographic CT. Eur Radiol 2003;13:1438-1443.

5. Carvalho GA, Nikkhah G, Matthies C, Penkert G, Samii M. Diagnosis of root avulsions in traumatic brachial plexus injuries: value of computerized tomography myelography and magnetic resonance imaging. J Neurosurg 1997;86:69-76.

6. Yoshikawa et al. Brachial plexus injury: Clinical manifestations, conventional imaging findings \& the latest imaging techniques. RadioGraphics 2006. 26:133-14.

7. Sureka J, Cherian RA, Alexander M, Thomas BP,(2009): MRI of brachial plexopathies. Clin Radiol. ;64(2):208-18.

8. Hems TE, Birch R, Carlstedt T. The role of magnetic resonance imaging in the management of traction injuries to the adult brachial plexus J Hand Surg [Br] 1999;24:550-555.

\section{Source of Support: Nil.}

Conflict of Interest: None Declared.

Copyright: (c) the author(s) and publisher. IJMRP is an official publication of Ibn Sina Academy of Medieval Medicine \& Sciences, registered in 2001 under Indian Trusts Act, 1882.

This is an open access article distributed under the terms of the Creative Commons Attribution Non-commercial License, which permits unrestricted non-commercial use, distribution, and reproduction in any medium, provided the original work is properly cited.

Cite this article as: Amit Disawal, Ashwini Bakde. Newer MR Imaging Techniques in Traumatic Brachial Plexopathies. Int J Med Res Prof. 2016; 2(5):233-37. 